\title{
Sistema de monitoreo electrónico de asfixia neonatal
}

\author{
Neonatal Asphyxia Electronic Monitoring System
}

\section{Sistema de monitoramento eletrônico de asfixia neonatal}

Jesús Arturo Hernández Soberón

Universidad Tecnológica del Norte de Coahuila, México

jasoberon@gmail.com

https://orcid.org/0000-0002-8116-6509

Miriam Zulema González Medrano

Universidad Tecnológica del Norte de Coahuila, México

zulema.gzz@hotmail.com

https://orcid.org/0000-0002-1962-2307

Raúl Zambrano Rangel

Universidad Tecnológica del Norte de Coahuila, México

raulzmbrn@gmail.com

https://orcid.org/0000-0001-8109-9580

\section{Resumen}

El síndrome de muerte súbita del lactante (SMSL) es una de las principales causas de muerte encontradas en las autopsias a bebés menores de un año. Aunque se han identificado múltiples factores de riesgo estadísticos, científicamente no hay certeza de qué provoca este trastorno. Por ello surge la idea de crear un dispositivo de monitoreo para medir las pulsaciones del lactante, capaz de emitir una alerta cuando se presente un problema respiratorio en bebés lactantes y así prevenir el SMSL. El método utilizado fue el del análisis. El dispositivo está compuesto por sensores piezoeléctricos, un microcontrolador, látex y cables. Para la producción del dispositivo, fue necesaria una máquina de prototipado de placa de circuitos impresos (PCB, por sus siglas en inglés). Con este proyecto se presenta una forma de monitorear los pulsos cardiacos del lactante: al detectar un ritmo 
diferente al normal, mediante el uso del internet de las cosas (IdC), emite de inmediato una notificación. Además, presenta tecnología open hardware y software de Arduino. Como se utiliza tecnología abierta, los usuarios tienen la posibilidad de mejorar el diseño o añadirle nuevas funcionalidades. Se trata de una muestra de que este tipo de tecnologías se pueden utilizar como herramientas de prevención en el campo de la medicina, en este caso, para prevenir la muerte súbita del lactante.

Palabras clave: dispositivo, lactante, monitoreo.

\section{Abstract}

Sudden infant death syndrome (SIDS) is one of the leading causes of death found at autopsies in infants under one year of age. Although multiple statistical risk factors have been identified, scientifically there is no certainty of what causes this disorder. Hence the idea to create a monitoring device to measure the pulsations of the infant, capable of emitting an alert when a respiratory problem occurs in nursing babies, and thus prevent SIDS. The method to be used is the analysis. The device is composed of piezoelectric sensors, a microcontroller, latex and cables. For the production of the device, a printed circuit board (PCB) prototyping machine was necessary. This project presents a way to monitor the infant's heart rate: detecting a different rhythm than normal, using the Internet of Things (IoT), immediately issues a notification. In addition, it features open hardware and Arduino software technology. Since open technology is used, this allows users to improve the design or add new functionalities. It is a sample that this type of technology can be used as prevention tools in the field of medicine, in this case, to prevent sudden infant death.

Keywords: device, infant, monitoring. 


\section{Resumo}

A síndrome da morte súbita do lactente (SMSL) é uma das principais causas de morte encontradas em autópsias de bebês menores de um ano de idade. Embora vários fatores de risco estatísticos tenham sido identificados, não é cientificamente certo o que causa esse transtorno. Por isso, surgiu a ideia de criar um dispositivo de monitoramento para medir a frequência cardíaca do bebê, capaz de emitir um alerta quando ocorre um problema respiratório em bebês amamentados e, assim, prevenir a SMSL. O método utilizado foi o de análise. O dispositivo é composto por sensores piezoelétricos, microcontrolador, látex e cabos. Para a produção do dispositivo, foi necessária uma máquina de prototipagem de placa de circuito impresso (PCB). Este projeto apresenta uma forma de monitorar os pulsos cardíacos do bebê: ao detectar um ritmo diferente do normal, por meio do uso da Internet das Coisas (IoT), ele emite imediatamente uma notificação. Além disso, possui tecnologia aberta de hardware e software do Arduino. Com a utilização de tecnologia aberta, os usuários têm a possibilidade de aprimorar o design ou adicionar novas funcionalidades a ele. Isso é um exemplo de que esse tipo de tecnologia pode ser utilizada como ferramenta de prevenção na área da medicina, neste caso, para prevenir a morte súbita de bebês.

Palavras-chave: dispositivo, bebê, monitoramento.

Fecha Recepción: Diciembre 2019

Fecha Aceptación: Junio 2020

\section{Introducción}

El síndrome de muerte súbita del lactante (SMSL) es la muerte de un bebé menor de un año de edad aparentemente saludable. Por lo general, sucede durante el sueño. A veces este trastorno se conoce como muerte de cuna porque, por lo general, como ya se mencionó, los lactantes mueren en sus cunas (Mayo Clinic, 5 de febrero de 2019.

Si bien la causa es desconocida, el SMSL podría estar relacionado con defectos en la porción del cerebro del bebé que controla la respiración y la vuelta al estado de vigilia.

De acuerdo con el portal Mayo Clinic (5 de febrero de 2019), expertos en el tema han descubierto algunos factores que podrían poner todavía en mayor riesgo a los bebés y han identificado algunas medidas para prevenir el SMSL. Entre estas últimas, tal vez la más importante, es colocar a tu bebé boca arriba para dormir. 


\section{Revista Iberoamericana de las Ciencias de la Salud}

Rachel Moon (2016) señala que la distinción entre SMSL y otras muertes súbitas e inesperadas en infantes (SUID, por sus siglas en inglés), particularmente aquellas que ocurren durante un período de sueño no observado (muertes infantiles relacionadas con el sueño), como la sofocación involuntaria, es desafiante, no puede determinarse por autopsia sola y puede permanecer sin resolver. Después de una investigación completa del caso, muchos de los factores de riesgo modificables y no modificables para el SMSL y la asfixia son sorprendentemente similares (Moon, 2016, p. 2).

Recientemente, la empresa Apno Systems ha desarrollado una muñequera que trata de prevenir las muertes súbitas y cuya principal misión es la de controlar el oxígeno y el ritmo cardiaco del bebé. El wearable, al que han bautizado como Infant Care System, en el caso de detectar alguna anomalía en las constantes vitales del menor, emite una alarma que llega directamente al smartphone de los padres para que puedan actuar con rapidez. Además, la muñequera emite una pequeña descarga en el cuerpo del bebé con el objetivo de recuperar la normalidad en los signos vitales del pequeño (Cid, 30 de marzo de 2016).

\section{Materiales y métodos}

\section{Detección de necesidad}

Los datos de los Centros para el Control y la Prevención de Enfermedades (CDC, por sus siglas en inglés) muestran que en 2010 (el año más reciente sobre el cual hay datos disponibles) murieron 2063 niños debido al SMSL, también conocido, por sus siglas en inglés, como SIDS (Murphy, Xu y Kochanek, 2013, citados en Oficina de Comunicaciones, 21 de enero de 2015)

El dispositivo en cuestión se comunicará vía wifi hacia una aplicación de smartphone, así se evitarán interrupciones entre los dispositivos. Además, tendrá un costo accesible para el mercado destinado. El producto está dirigido a padres de familia con hijos neonatales, propensos dentro de los primeros 11 meses de vida a sufrir de muerte de cuna. El diseño consta de un dispositivo de monitoreo para medir las pulsaciones del lactante y prevenir el SMSL.

Siguiendo a Medina (17 de septiembre de 2015), "los rápidos latidos del corazón de un bebé recién nacido son normales. Mientras la frecuencia de un adulto es de 60 a 80 latidos por minuto (lpm), la de los bebés suele ser entre 120 y 160 lpm” (párr. 4). Al mes de 
nacido, suele presentar de 100 a 150 lpm; a los dos años, de entre 85 y 125 lpm; a los cuatro años, de 75 a 115 lpm; a los seis años, de 65 a 100 lpm, y los mayores de seis años, de entre 60 y 100 lpm (Medina, 17 de septiembre de 2015). "El corazón de los pequeños late con más frecuencia porque es aún inmaduro" (Medina, 17 de septiembre de 2015, párr. 4).

El método a utilizar es el del análisis. El dispositivo está compuesto por sensores piezoeléctricos, un microcontrolador, látex y cables. En cuanto a los primeros, "el sensor piezoeléctrico puede estar conformado por materiales cerámicos o cristales iónicos que son capaces de generar una pequeña energía eléctrica cuando estos son deformados. A este efecto se le conoce como efecto piezoeléctrico" (Ingeniería Mecafenix, 3 de julio de 2018). Por lo que, para la producción del dispositivo, es necesaria una máquina de prototipado de placa de circuitos impresos (PCB, por sus siglas en inglés).

Para realizar el instrumento se utilizó lo siguiente:

1) Placa de desarrollo NodeMCU con chip wifi ESP8266.

2) Sensor de pulso cardiaco.

3) Buzzer.

4) Arduino IDE.

5) Blynk para móviles.

\section{NodeMCU ESP8266}

ESP8266 es el nombre de un microcontrolador diseñado por una compañía china llamada Espressif Systems, afincada en Shanghái. Su producción en masa data de principios del año 2014, cuando se dio a conocer que este chip sería una excelente solución, un puente para aquellos microcontroladores que requerían conectarse a una red inalámbrica wifi (Ceja, Rentería, Ruelas y Ochoa, 2017, pp. 24-36) (véase figura 1). 
Figura 1. NodeMCU ESP8266

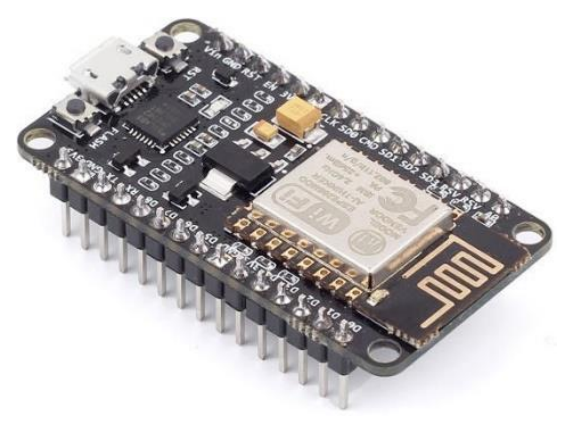

Fuente: 330ohms (s. f.)

\section{Sensor de pulso cardiaco}

El sensor de pulso amplifica la señal bruta del sensor de pulso anterior y normaliza la onda de pulso alrededor de V / 2 (punto medio en voltaje) respondiendo a los cambios relativos en la intensidad de la luz (Bolaños, 29 de junio de 2018, p. 3) (ver figura 2).

Figura 2. Sensor de pulso

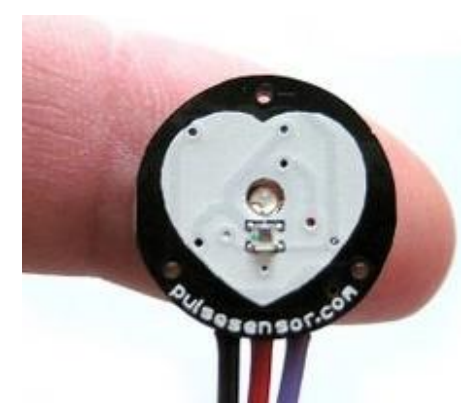

Fuente: World Famous Electronics llc. (s. f.)

En la tabla 1 se pueden observar las especificaciones de la conexión del sensor.

Tabla 1. Conexión del sensor

\begin{tabular}{|l|l|}
\hline NodeMCU & Sensor \\
\hline $3.3 \mathrm{~V}$ & + VCC \\
\hline GND & - GND \\
\hline A0 & S \\
\hline
\end{tabular}

Fuente: Elaboración propia 


\section{Arduino IDE}

El IDE de Arduino es un entorno de programación que ha sido empaquetado como un programa de aplicación; es decir, que consiste en un editor de código, un compilador, un depurador y un constructor de interfaz gráfica (GUI). Además incorpora las herramientas para cargar el programa ya compilado en la memoria flash del hardware (AprendiendoArduino, 2018)

\section{Buzzer}

Se trata de un aparato de señal eléctrica que hace que un zumbido de sonido (Merriam-Webster., s. f.) (Véase figura 3).

Figura 3. Buzzer

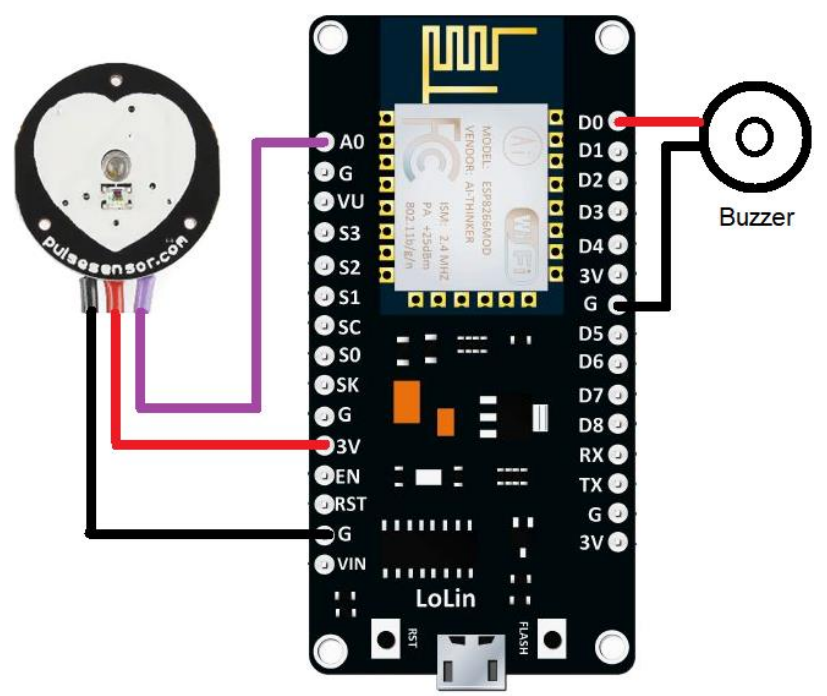

Fuente: Elaboración propia

\section{Blynk para móviles}

Es una plataforma de internet de las cosas (IdC) para Android y iOS de control de sistemas desarrollados con Arduino y Raspberry Pi (Tecnología Humanizada, 6 de noviembre de 2018). 
Muchas aplicaciones IdC requieren lo siguiente:

Almacenamiento masivo de datos, gran velocidad de procesamiento para permitir la toma de decisiones en tiempo real y redes de banda ancha de alta velocidad para transmitir datos, audio o video. La computación en la nube ofrece la solución ideal para el manejo de los flujos enormes de datos y su procesado para un número sin precedentes de dispositivos IoT y de seres humanos en tiempo real (Salazar y Silvestre, 2016, p. 25)

La arquitectura de funcionamiento se ilustra en la figura 4.

Figura 4. Blynk para móviles

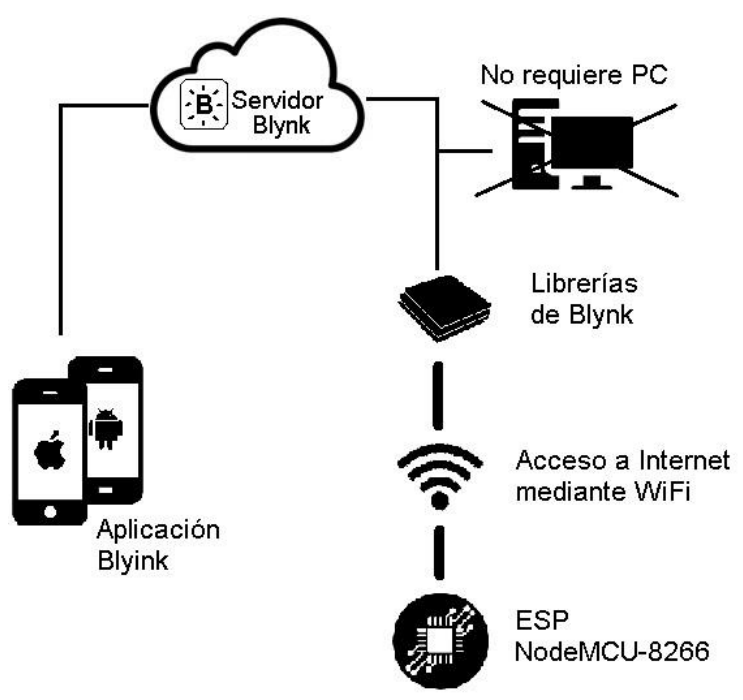

Fuente: Elaboración propia

El IdC crece y escala gracias a la tecnología o redes inalámbricas de sensores (WSN), y juega un papel importante en la comunicación de todas las redes, es decir, en la ubicuidad de estas (Cera, Mart, Rojas, Villaveces y Sanmart, 2015, p. 2).

\section{Código para la programación en el ESP8266 NodeMCU}

La programación se realizó con la estructura sketch de Arduino. En esta misma interfaz se agregan las librerías necesarias para poder trabajar la placa ESP8266, como si fuera en programación Arduino. Véase figura 5, 6 y 7. 
Figura 5. Librerías (parte uno)

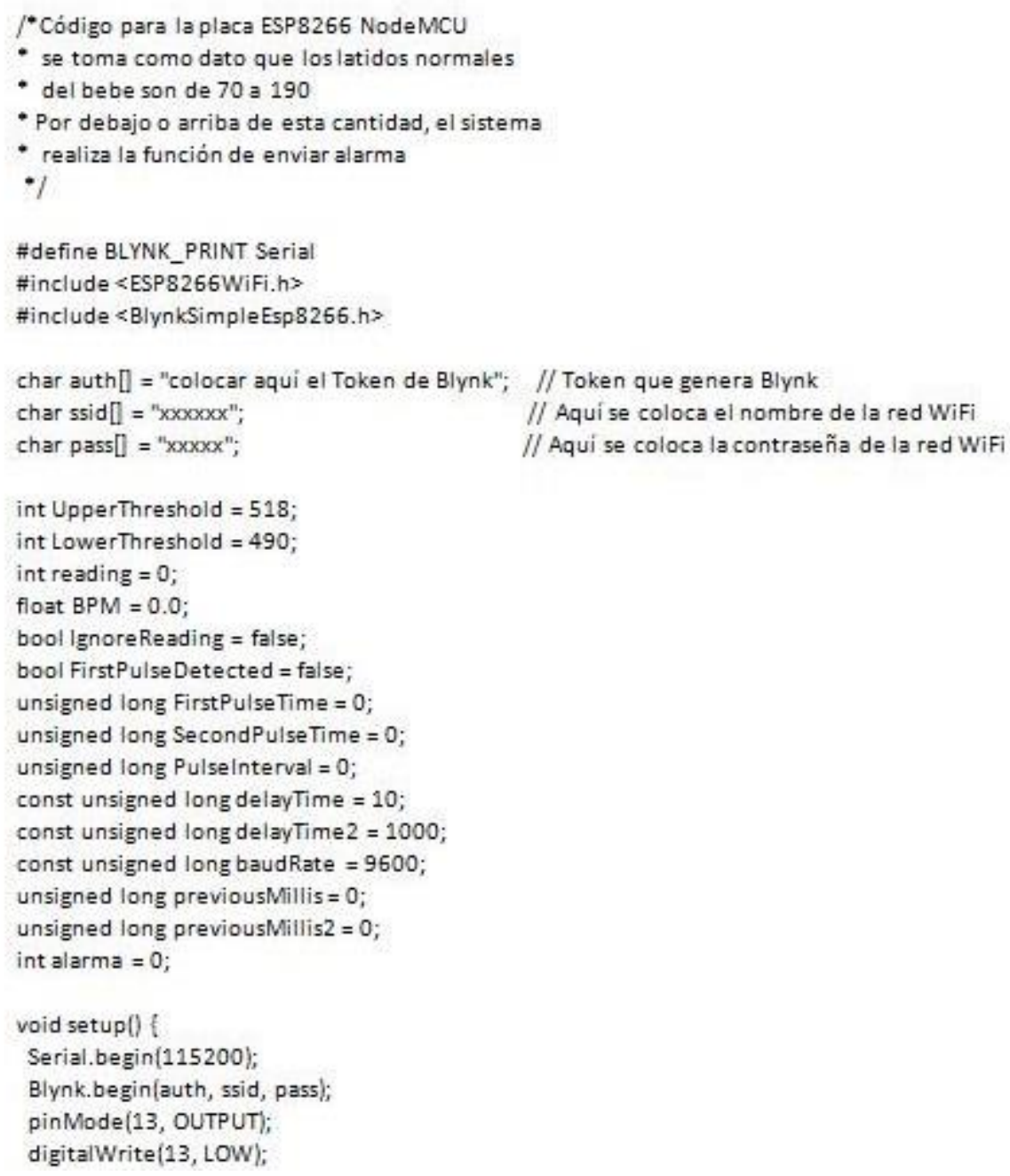

Fuente: Elaboración propia 
Figura 6. Librerías (parte dos)

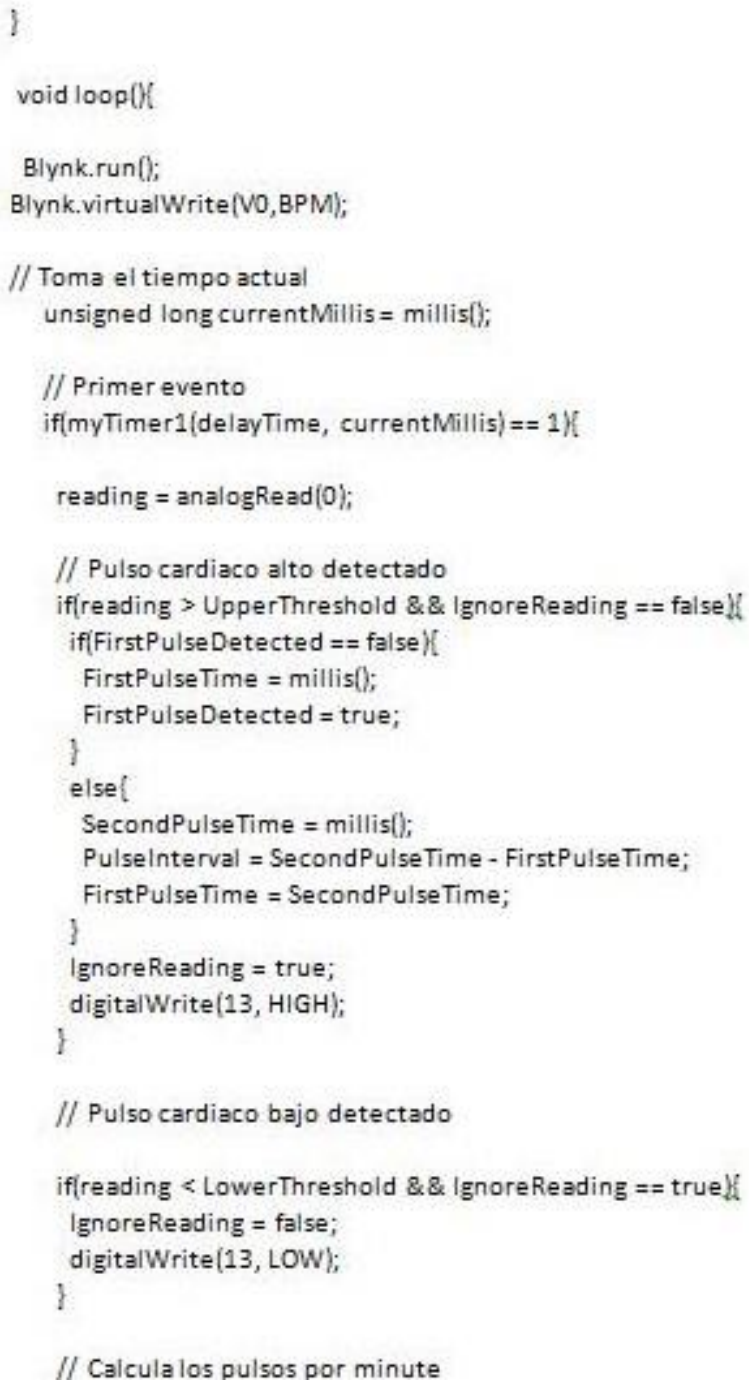

Fuente: Elaboración propia 
Figura 7. Librerías (parte tres)

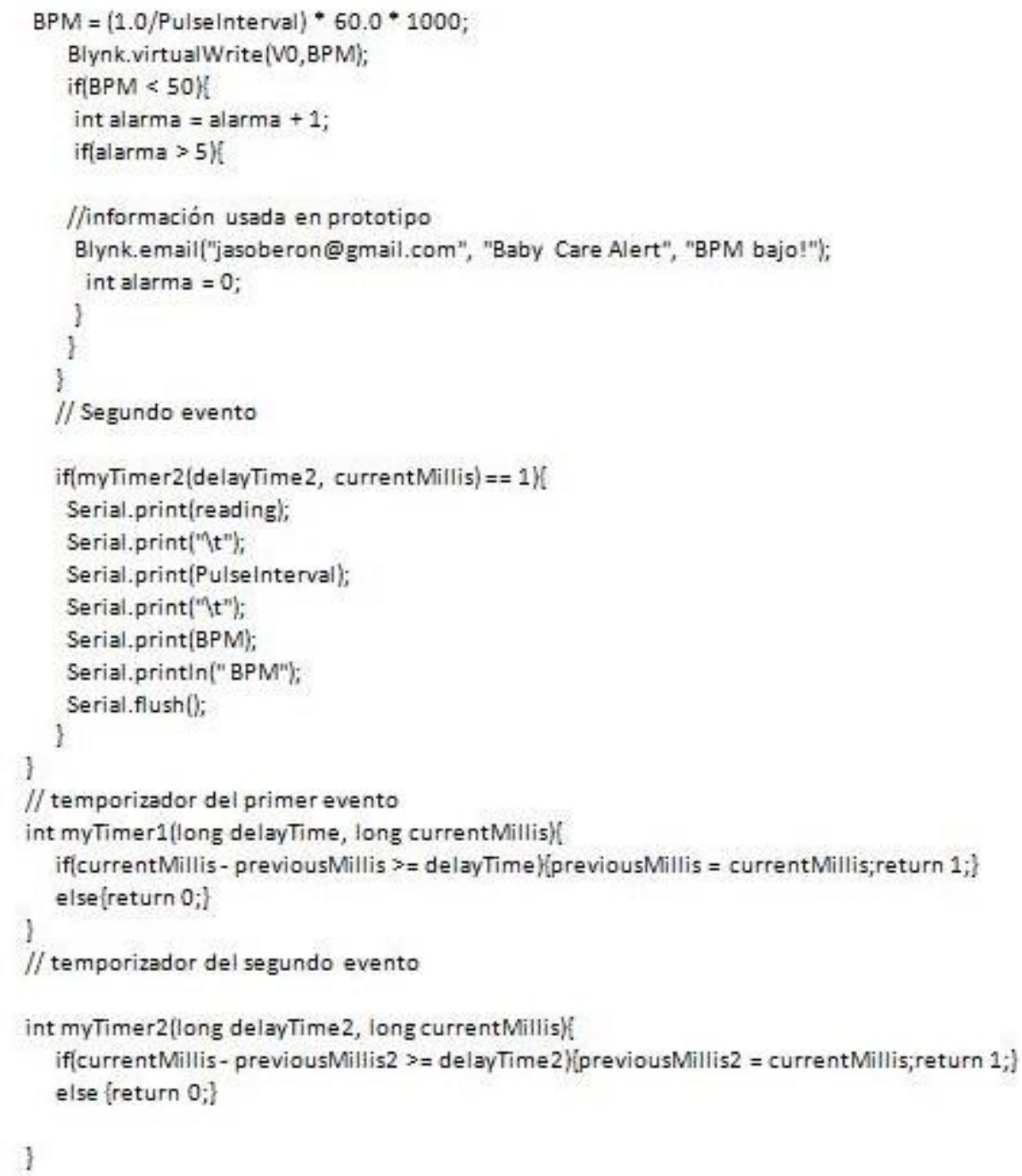

Fuente: Elaboración propia

\section{Configuración de la aplicación en Blynk}

A continuación se muestran los pasos a seguir para la configuración de la aplicación en un celular utilizando Blynk, que servirá como parte visual de monitoreo del dispositivo. (Véase figura 8 y 9) 
Figura 8. Vistas Android

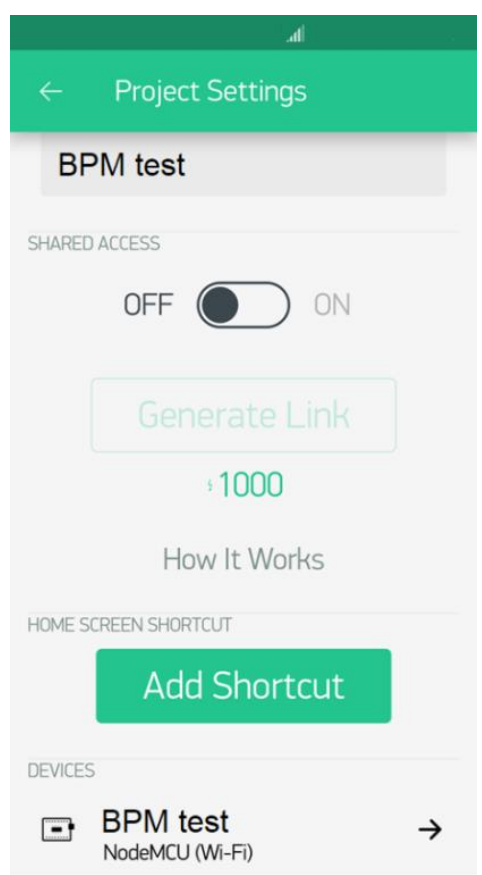

Fuente: Elaboración propia

Figura 9. Vistas Android

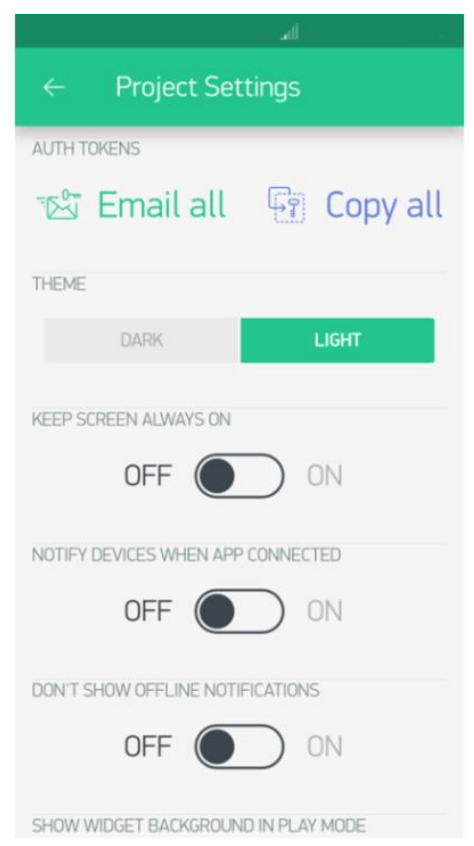

Fuente: Elaboración propia 


\section{Indicadores}

En este punto, seleccionamos el indicador Gauge. Ver figura 10.

Figura 10. Indicadores

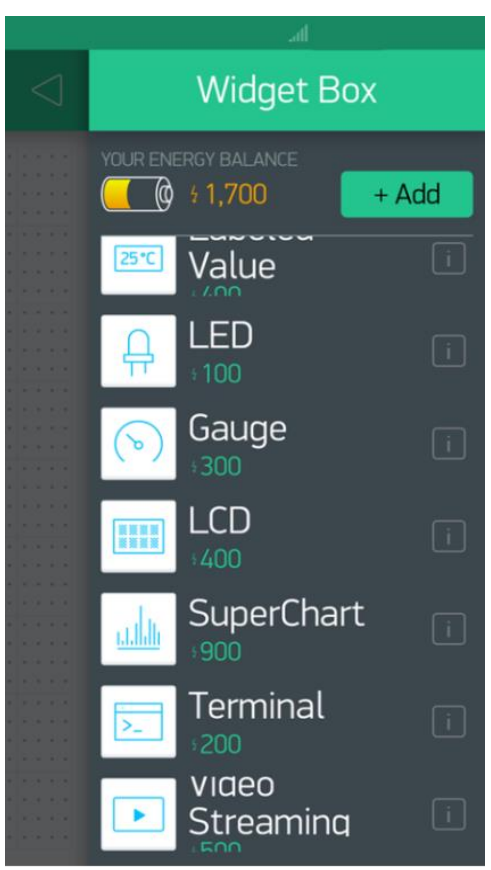

Fuente: Elaboración propia

Tablero en modo edición. Organización y configuración del indicador

En la figura 11 y 12 se muestra la configuración del indicador en la aplicación. 
Figura 11. Configuración del indicador en la aplicación

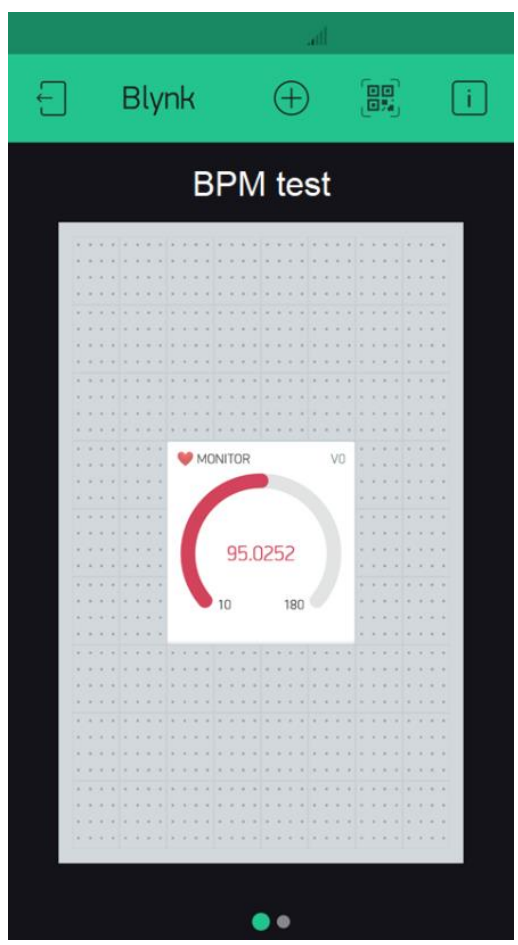

Fuente: Elaboración propia

Figura 12. Vistas Android

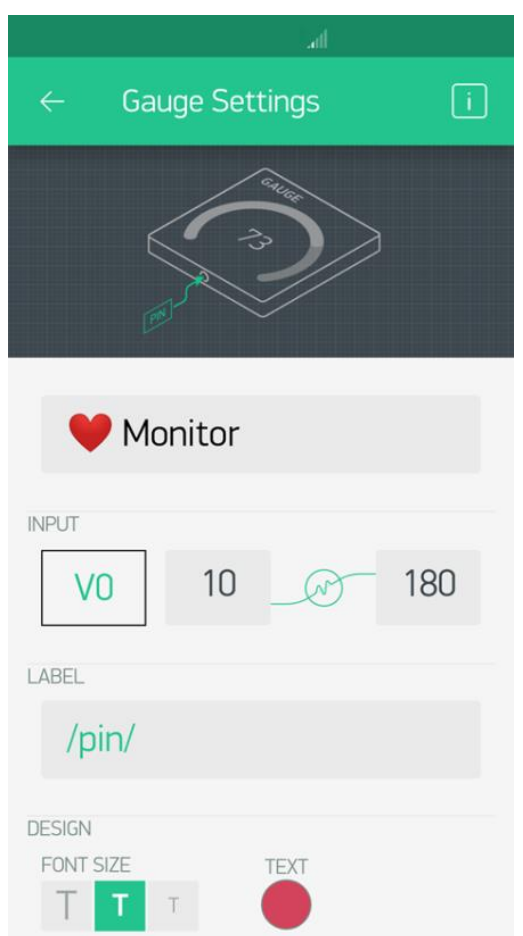

Fuente: Elaboración propia 
El indicador ADC0 realizará la lectura dada del acondicionamiento de la señal del sensor de pulsaciones por minuto (BPM, por sus siglas en inglés). La configuración se coloca a escala de 0 a 200 puntos, con una actualización en tiempo real de dos segundos por muestreo.

\section{Tablero visual funcionando en un celular con la aplicación configurada en Blynk}

A continuación, se muestra en la figura 13 y 14 la presentación final de la aplicación en Blynk

Figura 53. Tablero visual en línea

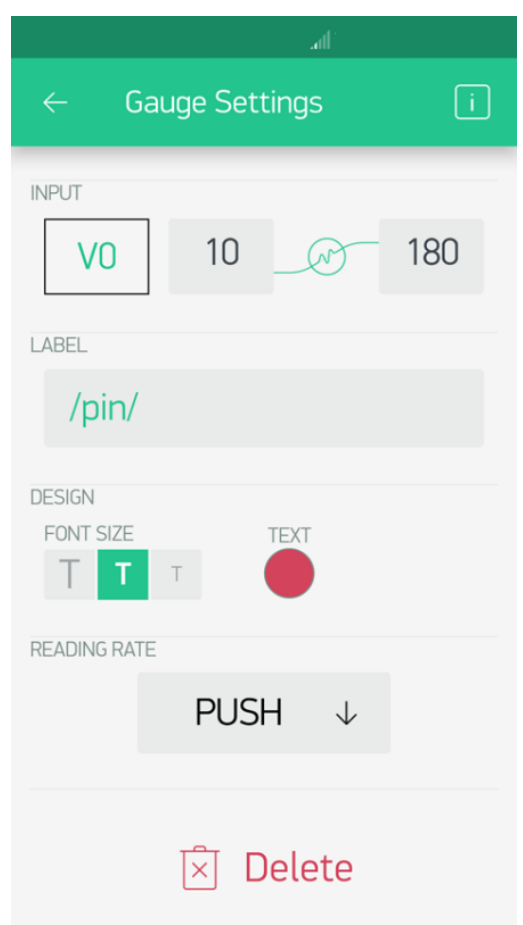

Fuente: Elaboración propia

El teléfono celular, de cualquier marca, es ahora nuestro tablero de control y el medio para una administración inteligente de la energía y el aumento de vida útil de equipos (Chávez y Vega, 14 de agosto de 2019). 
Figura 64. Dashboard en línea

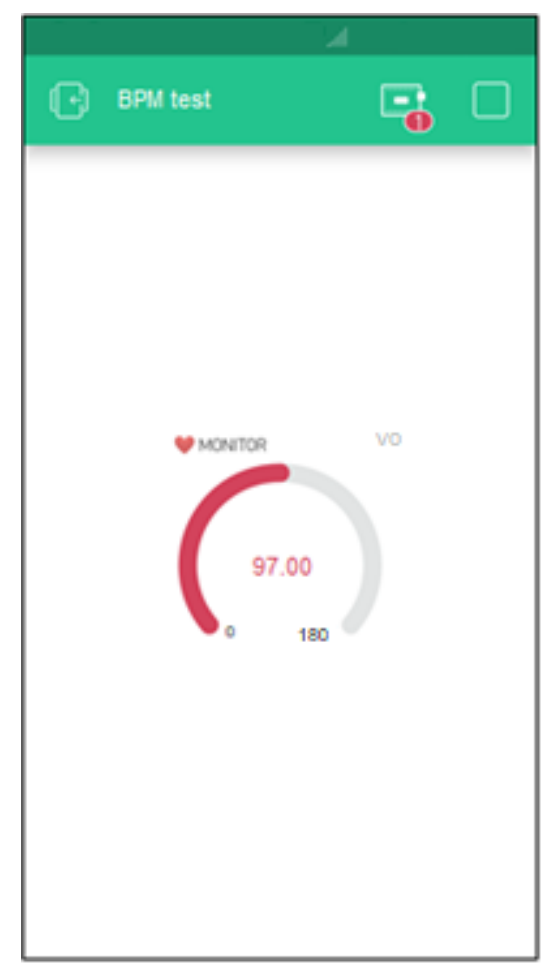

Fuente: Elaboración propia

\section{Estudio financiero}

La tasa interna de retorno (TIR) es la tasa de interés o rentabilidad que genera un proyecto (Restrepo, 2017). Se provee con una inversión de 5000 pesos mexicanos. El costo del dispositivo prototipo es de 300 pesos. El costo de producción para cinco dispositivos ensamblados en una sola placa utilizando solamente un pin de entrada de la placa ESP8266 y acoplado el sensor BPM es de aproximadamente 2000 pesos en una compañía externa dedicada a esta fabricación. Para una producción mayor, el precio disminuye. La cantidad de ventas que deberían realizarse para recuperar el coste de inversión será de al menos cuatro dispositivos. La relación de capital proviene de una inversión propia.

Las estrategias de fijación de precio para el dispositivo son del tipo incrementado, establece un costo base por encima del costo de producción. El costo estará ajustado con respecto al costo de la competencia. 


\section{Resultados}

Con una placa Arduino Uno se programaron las simulaciones de pulsos cardiacos en forma de datos analógicos que van desde los valores de 10 a 180 pulsos por minuto. Estos datos se enviaron en forma de comunicación serial entre el Arduino Uno utilizado como simulador y el prototipo usando la placa ESP8266 NodeMCU. Como primera prueba se realizaron secuencias simuladas de pulsos cardiacos normales por un período de cinco minutos continuos. Se observó que el prototipo responde de forma correcta en tiempo real; solo al enviar los datos a la nube, a través de la aplicación Blynk, se observa un retraso que varía desde uno a dos segundos en mostrar la información. En una segunda prueba, se generó una secuencia normal de pulsos cardiacos por un minuto para luego enviar pulsos por debajo y alto de lo normal que se pudieran presentar en los bebés. La respuesta fue satisfactoria, ya que se logró encender la alarma del prototipo, así como la señal de alarma enviada a través de la nube mediante la aplicación de Blynk.

Con esta información logramos demostrar el funcionamiento correcto. Aunque existe el tema del retardo en comunicación fuera del dispositivo, este no afecta, ya que la alarma se activa de forma local, y la alarma al dispositivo remoto se suscita con aproximadamente un segundo de retardo.

Una mejora que se puede realizar a futuro es la de reprogramar utilizando inteligencia artificial. Esto debido a que se pudieran presentar falsas alarmas con algún movimiento brusco del bebé, al dar una patada, al darse vuelta o al experimentar algún cambio en sus signos vitales que no sean inofensivos.

\section{Discusión}

La innovación que se está llevando a cabo es incremental, debido a que ya existen otras versiones muy parecidas, como el que ofrece la empresa Apno Systems. El prototipo que se presenta es funcional y es fácil de interpretar por los papás: en cuanto se presenta un problema que puede comprometer la salud del bebé y deba ser atendido lo más pronto posible. Aunado a que está adecuado para que sea accesible en el mercado en el que se introducirá (presentación tipo wearable más económico). El nivel en el que se encuentra el prototipo actualmente es disociador debido a que las innovaciones con las que cuenta están en algunos de sus componentes. El producto es viable ya que presenta una solución económica que nadie ofrece en nuestra región ante este síndrome. 


\section{Conclusiones}

Con este proyecto se presenta una forma de monitorear los pulsos cardiacos del lactante: al detectar un ritmo diferente al normal, mediante el uso del IdC, emite de inmediato una notificación. Además, se presenta con tecnología open hardware y software de Arduino. Como se utiliza tecnología abierta, los usuarios tienen la posibilidad de mejorar el diseño o añadirle nuevas funcionalidades al mismo. Se trata de una muestra de que este tipo de tecnologías se pueden utilizar como herramientas de prevención en el campo de la medicina, en este caso, para prevenir la muerte súbita del lactante.

\section{Referencias}

330ohms. (s. f.). NodeMCU ESP8266 ESP-12 WiFi IoT. 330ohms. Recuperado de https://www.330ohms.com/products/esp8266-esp-12-nodemcu-lua-wifi-iot.

AprendiendoArduino. (9 de abril de 2018). Entender Arduino. AprendiendoArduino. Recuperado de https://aprendiendoarduino.wordpress.com/tag/ide/.

Bolaños, D. (29 de junio de 2018). Sensor de pulso cardiaco. Recuperado de http://www.bolanosdj.com.ar/MOVIL/ARDUINO2/TeoriaSensorPulsos.pdf.

Ceja, J., Rentería, R., Ruelas, R. y Ochoa, G. (2017). Módulo ESP8266 y sus aplicaciones en el internet de las cosas. Revista de Ingeniería Eléctrica, 1(2), 24-36.

Cera, J., Mart, L. M., Rojas, J., Villaveces, J. y Sanmart, P. (2015). Apoyo al estado del arte del internet de las cosas en salud. Investigación y Desarrollo en TIC, 6(1), 2.

Cid, R. F. (30 de marzo de 2016). Un wearable para evitar la muerte súbita de los bebés. socialmediatica. Recuperado de https://socialmediatica.com/wearable-evitar-lamuerte-subita-los-bebes/.

Chávez, I. y Vega, Y. (14 de agosto de 2019). Interruptor de potencia y tablero de control desde el celular. Energía Hoy. Recuperado de https://energiahoy.com/2019/08/14/interruptor-de-potencia-y-tablero-de-controldesde-el-celular/.

Ingeniería Mecafenix. (3 de julio de 2018). Sensor piezoeléctrico (efecto piezoeléctrico). Ingeniería Mecafenix. La enciclopedia de la ingeniería. Recuperado de https://www.ingmecafenix.com/automatizacion/sensores/sensor-piezoelectrico/. 
Mayo Clinic. (5 de febrero de 2019). Síndrome de muerte súbita del lactante (SMSL). Mayo Clinic. Recuperado de https://www.mayoclinic.org/es-es/diseasesconditions/sudden-infant-death-syndrome/symptoms-causes/syc-20352800.

Medina, V. (17 de septiembre de 2015). El rápido latido del corazón de un bebé. guiainfantil.com. Recuperado de https://www.guiainfantil.com/blog/539/el-rapidolatido-del-corazon-de-un-bebe.html.

Merriam-Webster. (s. f.). buzzer. Merriam-Webster. Retrieved from https://www.merriamwebster.com/dictionary/buzzer.

Moon, R. Y. (2016). SIDS and Other Sleep-Related Infant Deaths: Evidence Base for 2016 Updated Recommendations for a Safe Infant Sleeping Environment. Pediatrics, 138(5). Retrieved from https://pubmed.ncbi.nlm.nih.gov/27940805/.

Oficina de Comunicaciones. (21 de enero de 2015). ¿Cuántos bebés mueren a causa del síndrome de muerte súbita del lactante (SIDS por sus siglas en inglés) o corren riesgo de tener el SIDS? Instituto Nacional de la Salud infantil y Desarrollo Humano Eunice Kennedy Shriver. Recuperado de https://espanol.nichd.nih.gov/salud/temas/sids/informacion/bebes\#f1.

Restrepo, M. (23 de 10 de 2017). Rankia. Obtenido de https://www.rankia.co/blog/mejorescdts/3718561-que-tir-para-sirve

Salazar, J. y Silvestre, S. (2016). Internet de las cosas. Praga, República Checa: TechPedia. Recuperado de https://upcommons.upc.edu/bitstream/handle/2117/100921/LM08_R_ES.pdf.

Tecnología Humanizada. (6 de noviembre de 2018). Blynk, plataforma de internet de las cosas en la red. Tecnología Humanizada. Recuperado de https://humanizationoftechnology.com/blynk-plataforma-de-internet-de-las-cosasen-la-red/revista/2018/volumen-4-2018/11/2018/.

World Famous Electronics 1lc. (s. f.). Pulse Sensor. World Famous Electronics 1lc. Retrieved from https://pulsesensor.com/. 


\begin{tabular}{|l|l|}
\hline Rol de Contribución & Autor (es) \\
\hline Conceptualización & Jesús Arturo Hernández Soberón \\
\hline Metodología & $\begin{array}{l}\text { Jesús Arturo Hernández Soberón «igual» } \\
\text { Miriam Zulema González Medrano «igual» } \\
\text { Raúl Zambrano Rangel «igual» }\end{array}$ \\
\hline Software & Jesús Arturo Hernández Soberón \\
\hline Validación & Jesús Arturo Hernández Soberón \\
\hline Análisis Formal & Jesús Arturo Hernández Soberón \\
\hline Investigación & $\begin{array}{l}\text { Jesús Arturo Hernández Soberón «igual» } \\
\text { Miriam Zulema González Medrano «igual» }\end{array}$ \\
\hline Recursos & Jesús Arturo Hernández Soberón \\
\hline Curación de datos & $\begin{array}{l}\text { Jesús Arturo Hernández Soberón «igual» } \\
\text { Miriam Zulema González Medrano «igual» }\end{array}$ \\
\hline $\begin{array}{l}\text { Escritura - Preparación del } \\
\text { borrador original }\end{array}$ & Miriam Zulema González Medrano \\
\hline $\begin{array}{l}\text { Escritura - Revisión y } \\
\text { edición }\end{array}$ & $\begin{array}{l}\text { Jesús Arturo Hernández Soberón «igual» } \\
\text { Miriam Zulema González Medrano «igual» }\end{array}$ \\
\hline Visualización & $\begin{array}{l}\text { Jesús Arturo Hernández Soberón «igual» } \\
\text { Miriam Zulema González Medrano «igual» }\end{array}$ \\
\hline Supervisión & Miriam Zulema González Medrano \\
\hline $\begin{array}{l}\text { Administración de } \\
\text { Proyectos }\end{array}$ & $\begin{array}{l}\text { Jesús Arturo Hernández Soberón «igual» } \\
\text { Miriam Zulema González Medrano «igual» }\end{array}$ \\
\hline Adquisición de fondos & $\begin{array}{l}\text { Jesús Arturo Hernández Soberón «igual» } \\
\text { Miriam Zulema González Medrano «igual» } \\
\text { Raúl Zambrano Rangel «igual» }\end{array}$ \\
\hline
\end{tabular}

